\title{
DESIGNING A ROADMAP FRAMEWORK FOR HEALTHCARE SYSTEMS ADOPTION
}

\author{
Sinte Mutelo ${ }^{1}$ and Suama Hamunyela ${ }^{2}$ \\ ${ }^{1} \mathrm{Ms}$. \\ ${ }^{2} \mathrm{Dr}$. \\ Namibia University of Science and Technology \\ 13 Jackson Kaujeua Street, Windhoek, Namibia
}

\begin{abstract}
Developing country healthcare industry have policies and procedure to regulate health information systems (HIS) both in private and public sector. The Healthcare institutions as part of the government with the help of Non-Governmental Organisation (NGOs) have implemented information systems (IS) within its different directorates to maximise the quality of care. However, HIS benefits may not be recognised by the healthcare organisation and stakeholders when systems implementation and adoption of policies and procedures are not backed up by roadmap to archive HIS goals. Thus, the need for healthcare roadmap to guide the development of HIS is important to the future development of a product. A roadmap development needs different stakeholders to participate. Therefore, this study, propose a roadmap that will provide guide for planning, selection, implementation, and adoption of HIS to produce quality healthcare services in Namibia. The study adopted a qualitative research method, because of its suitability to assist the research answer the research questions in order to meet the research objectives. Data was collected using three different techniques, semi-interviews, documents analysis and questionnaire. A questionnaire was used to collect data about the applicability, usability, and the importance of roadmapping. Then data collected was interpretively analysed. Based on the interpretations and the findings from the analysis of data, the healthcare industry roadmap was developed.
\end{abstract}

\section{KEYWORDS}

Roadmap, Healthcare, Health information systems (HIS), Adoption

\section{INTRODUCTION}

Healthcare industry is slow to adopt technology compared to other industries, due to a number of reasons including lack of HIS experts to implement and to utilise the technology. Health information systems is the planned delivery of health services to individuals by healthcare professionals (Beam, \& Kohane, 2018).

Health Information Systems (HIS) are interrelated systems designed to manage healthcare data, these handle data related to activities of providers and health firms (Sayles (2012). The systems with integrated efforts, maximise advantage to improve patient results, inform research, and influence policy-makers and decision-making stakeholders (Andargoli, Scheepers, Rajendran, \& Sohal, 2017). In most developing countries, the Ministry of Health is responsible for implementing the National Health Information System (NHIS) but health information systems are under-utilised. HIS assist healthcare to gather, process and disseminate information within the firm and their stakeholders (Wang, Kung, \& Byrd, (2018). Therefore, adopting of HIS is a challenge facing the health sector in developing countries as a result of technology changes, system implementation issues, and isolated systems. A roadmap towards HIS adoption is a proposed answer to alleviate the challenge and enforce the use of HIS in healthcare for quality data (Cetindamar, Phaal \& Probert, 2016).

This means that if it is properly developed, a roadmap will support sustainable ICT implementation. Technology roadmap offers power and flexibility in terms of technology implementation (Zamberlan, 2015). The roadmaps have been used to guide focus the organisation strategic plans efficiently. It assists various stakeholders to reach organisational goals. In addition, roadmapping is the most commonly used technique for supporting the strategic management of technology. 
Renals, et al., 2014 stated that "roadmapping is a process to communicate the development and integration of science and technology, and their incorporation into products or services to address business or societal opportunities" (p.41).

Therefore, roadmapping provides important details that determine the technical requirements and new ways of research and development.

Furthermore, a roadmap stipulates opportunities in ways to enlighten research groups of the upcoming and re-engineering the clinical research technologies (Managements, 2014). Implementation and system restructuring involving all stakeholders are important for changing the healthcare systems and improving health quality in the country (Jin, Jeong \& Yoon, 2015).

Despite a variety of efforts to embrace technologies within the health sector, adoption and usage of Health Information systems in developing healthcare industry remain problematic without strategic plans, policies to determine current, present and future technologies.

Thus, a roadmap towards HIS adoption is a solution to alleviate some of the challenges and enforce the use of HIS in health for quality healthcare. The purpose of this paper is to identify the importance of roadmap for improving the quality of healthcare when applied organisation.

The objective of this paper is to develop a roadmap towards HIS adoption in the health that can guide the health sector delivery quality services. The roadmap will provide a guide for planning, selection, implementation, and adoption of HIS to produce quality healthcare services in any context. This paper provides view of HIS stakeholders and roadmap experts on the HIS adoption roadmap. The main question addressed in this paper is: How can a Health Information system adoption roadmap be developed to enhance the quality of health?

\section{OVERVIEW OF HEALTH INFORMATION SYSTEMIN HELATHCARE}

Despite a variety of efforts to embrace technologies within the health sector, adoption and usage of Health Information Systems in developing countries is ongoing. Studies show a positive implementation of HIS has been ongoing, impacting the health service delivery in some developing countries (Karon, 2015). Ongundaini (2016) claims that there are limitations in terms of usability, functionality and peculiar social-technical factor; these systems are not user-friendly and not integrated. Most existing implementation of HIS techniques does not support or enhance quality healthcare services.

The developing countries health sector for example has no clear roadmap for HIS adoption. Though the health industry responsible for healthcare services has gone on board to accelerate the HIS process under the founded stakeholders, there is still no significant change in terms of HIS adoption. The absence of HIS strategic plan or policies may hinder HIS adoption. One of the assessments done in the health industry found that "much of the effort to date has been uncoordinated and directed mostly to isolated technical aspects of the HIS and not to institutional, coordination, and other behavioural aspects" (Khan \& Edwards, 2012, p.15).

Furthermore, the report stipulated that "there is as yet no mechanism to effectively engage the leadership of the Healthcare-A and other national agencies in this process, and there is currently no up-to-date HIS strategic plan or policy" (Khan \& Edwards, 2012, p.15). To deal with the complexity of HIS adoption concrete planning approach must be in place, a systematic approach that guide the health organisation to establish their HIS objectives and translate them to functional and technical requirements as well as identification of resources to implement HIS. Thus this study propose the components for consideration in the development of healthcare roadmap.

\section{LITERATURE REVIEW}

A roadmap is a plan that enables organisation to be able to align its technologies and business goals (Cetindamar, Phaal, \& Probert, 2016).

Roadmapping (TRM) has been widely used as a strategic management device to help governments and private sectors in effectively recognising potential services for the future, determining proper technology alternatives and mapping them with resource allocation plans (Carvalho, Fleury \& Lopes, 2013). Aleina, 
Viola, Fusaro, and Saccoccia (2017) argued that technology planning is important for decision makers considering the competitive problems that many organisations are facing.

Literature review revealed that for HIS adoption to take place in healthcare organisations, the organisations needs support from higher decision bodies such as its government that support by allocation sufficient resources and employing skilled professionals. Stakeholder involvement is one critical part when considering system development, adoption and implementation.

Accordingly Piña, Cohen, Larson, Marion, Sills, Solberg and Zerzan (2015) said that healthcare system needs to be redesigned, and that huge task must be composed with key healthcare stakeholders including doctors, nurses, administrators, health information managers and policymakers, and as well as with patients. For the functionality of HIS, an information systems, need all IS components complete. Sayles (2012) specified "an IS consists of data, people, and work processes and a combination of hardware (machines and media), software (computer programs), and communications technology (computer networks) known as information technology (IT)" (p.850).

Based on the papers reviewed, the most important factors affecting the adoption of HIS in the health sector are governance, stakeholder involvement, IT skills, IS Infrastructure.

Different technology adoption model were reviewed by the research. The following theories were noted; Innovation Diffusion Theory, technology acceptance model (TAM), Concerns-Based Adoption Model (CBAM), unified theory of acceptance and use of technology (UTAUT) and DeLone and McLean IS success Model.

Rogers' Innovation Diffusion Theory, one of the most popular theories for studying adoption of information technologies (IT) and understanding how IT innovations blow-out inside and amongst communities. To Zhang, Yu, Yan, and Spil (2015) this theory, innovation is an idea, process, or a technology that is perceived as new or unfamiliar to individuals within a particular area or social system.

Atkin, Hunt, and Lin, (2015) debated that "the technology acceptance model is a widely used theoretical framework originating from information sciences to conceptualize technology adoption. The model provides an ungenerous and straightforward approach to studying how users accept and use a technology" (p.631).

On the hand, the concern-based model, the model finds and provides ways to assess seven stages of concern. Haines (2018) in this article noted, one model for change in individuals is the Concerns-Based Adoption Model, it applies to anyone experiencing change, that is, policy makers, teachers, parents even students. The UTAUT suggests that four core constructs (performance expectancy, effort expectancy, social influence and facilitating conditions) are direct elements of behavioural intention and ultimately behaviour, and that these constructs are in turn moderated by gender, age, experience, and voluntariness of use (Williams, Rana, \& Dwivedi, 2015).

On the other hand, information systems success model (DeLone and McLean IS success model) theory which provides a comprehensive understanding of IS success by identifying, describing, and explaining the relationships among six of the most critical dimensions of success along which information systems are commonly evaluated. IS success model is considered one most powerful theories in contemporary information systems research. These models have been researched and found fit to other situation but not in this case.

\section{METHODOLOGY}

The study adopted a qualitative research method, because of its suitability to assist the research answer the research questions in order to meet the research objectives. To Silverman (2013), the reason for using the qualitative method is that the method is particularly important in the behavioural sciences where the aim is to realise the underlying motives of human behaviour.

Healthcare-A is a case studied in this paper. Mutelo and Jere (2017) defined case study as a tool of examination found in several field, in which research brings out an in depth analysis of the case. Hesse-Biber and Leavy (2011) pointed out that the uniqueness of a case study approach is that it provides the researcher with a complete understanding of a phenomenon within its social context.

Data was collected through three different techniques, semi-interviews, documents analysis and questionnaire. Questionnaires, documents, and interviews were used to collect data about the case studies. The interviewees involved systems users, top managers, and HIS implementers from the organisations. This 
was to ensure that interviewees were knowledgeable and skilled in discussing the areas pertaining to their positions. During the interviews, the researcher used a predefined set of questions. These sets of questions were of significance to the researcher as they were used as a guide for sustaining consistency of the questions asked to the interviewee. A questionnaire was used to obtain qualitative data about the applicability, usability, and knowledge of developing a technology roadmap from different HIS and IT roadmap experts.

\section{RESULTS}

\subsection{Interview Findings}

Healthcare-A will not function properly without policies to govern and manage its activities in achieving its planned goals and objectives. Without policies and strategic plans to guide and regulate the use of HIS, the Healthcare-A would have various types of HIS not needed for their business processes.

The policies regarding IT in Healthcare-A were formulated and developed by the IT department at the government level together with the IT directors and other management from the various government offices. According to one of the participants within Healthcare sector A mentioned, it is a good idea developing a roadmap for HIS adoption for the healthcare industry, because it will help align all plans within the organisation.

Therefore, one can conclude to say users of HIS have the interest to adopt HIS, but are held backward by existing processes and policies and a lack guide. This showed the support of employees

\subsection{Questionnaire Findings}

\subsubsection{Health Information System Adoption Roadmap Development Stakeholder}

Experts who were engaged in the research were asked to determine who should be part of the development of the HIS adoption roadmap. Five categories of some of the stakeholders were given to choose from: Policy makers; HIS users; HIS software developers; the entire Healthcare-A's directorates and health practitioners.

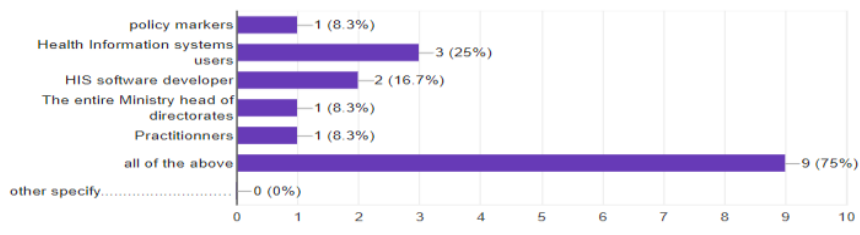

Figure 1. HEALTH information system adoption roadmap development and stakeholder

The questionnaire data shows the categories of stakeholders who can be part of the HIS development team. Most respondents choose all categories.

\subsubsection{The Relationship between HIS Roadmap and Quality Healthcare (QH)}

Health Information System roadmap aims to improve the healthcare services activities and processes in the country. The healthcare sector is very sensitive by nature as it involves people's lives and any errors can lead to serious damages such as loss of life. Thus, the HIS roadmap can be viewed as an approach that can guide the policy development and implementation across the country healthcare services. In turn, the policies developed to guide the healthcare professionals, patients, and management on how they should interact in the provision of quality healthcare services. As a result, there is a link between effective HIS roadmap and quality healthcare services. According to one of the respondents that was in support of an effective HIS roadmap, they stated that;

"A good HIS roadmap facilitates HIS technology adoption and use, which in turn contributes to the provision of quality healthcare" (RS2, pg7, 18). 
The findings clearly shows that there is a link between healthcare services and HIS roadmap is an indication that quality healthcare services are not offered in isolation, but rather there is a need for the integration of various components such as technologies and people.

\subsubsection{Technologies to Enhance Quality Health}

Figure 2. shows the different technologies used to enhance quality health to sustain the implemented HIS in the health sector of Healthcare-A in developing country.

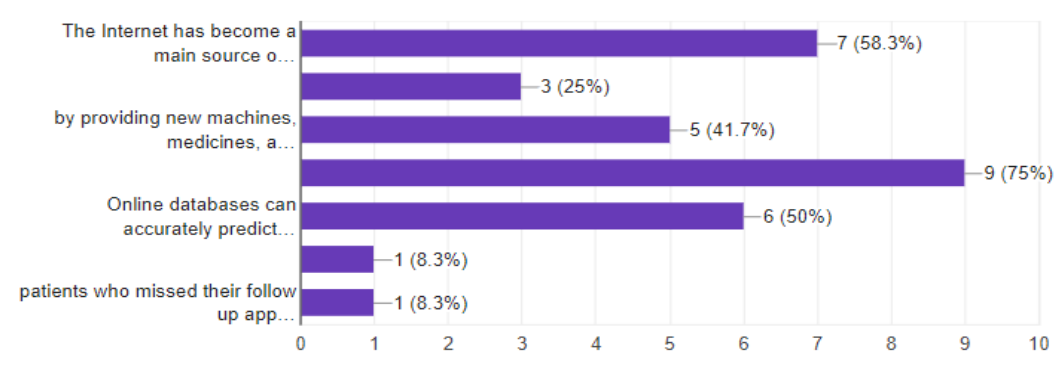

Figure 2. Technologies to enhance Quality Health

Experts and stakeholders of Healthcare-A have different knowledge and experience in information technology. Since the stakeholders are from different specialisations, this question was asked to find out the different technologies which could best suit the utilisation of HIS or are in use in the Healthcare-A to enhance quality health.

\subsubsection{General Understanding of Technology Roadmap by Experts}

The reason for studying the understanding of technology roadmap within the healthcare institutions and its stakeholders is to measure the level of expert knowledge. Figure 3 shows in detail that the term technology roadmap is not a very well knowledge strand within the expert Healthcare-A stakeholder. Employees and stakeholders, IT experts of Healthcare-A had different levels of technology roadmap understanding. Participants understanding was informed by their area of specialisation in the field of IT.

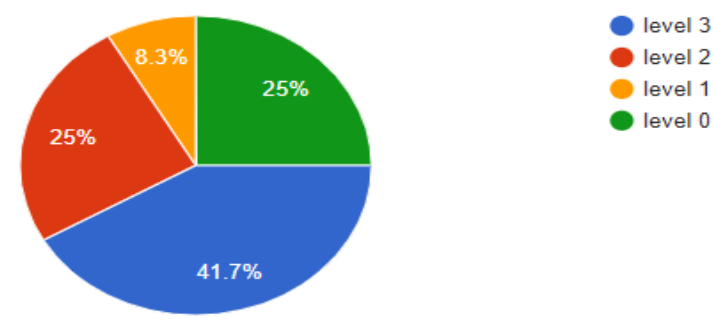

Figure 3. Technology roadmap level of understanding

To assess respondents' level of understanding four (4) categories level were created as follows. The results show that a big number of experts know what a TRM is and could define technology roadmap it.

\subsubsection{Roadmap Development for the Developing Healthcare}

The main objective of this research is to showcase a proposed HIS adoption Roadmap framework for healthcare institutions in order to have quality healthcare. Relevance of a roadmap framework was again confirmed in findings from questionnaires, where majority of respondents indicated that it is relevant.

Therefore, the majority feels it is important to develop a roadmap for the Healthcare-A. 


\subsubsection{The Relationship between Health Information System Roadmap and Quality Health}

HIS Roadmap aims to improve the healthcare services activities and processes in the country. The healthcare sector is very sensitive by nature as it involves people's lives and any errors can lead to serious damages such as loss of life. Thus, the HIS roadmap can be viewed as an approach that can guide the policy development and implementation across the healthcare industries. Result show that the link between healthcare services and HIS roadmap is an indication that quality healthcare services are not offered in isolation, but rather there is a need for the integration of various components such as technologies and people.

\section{ROADMAP DESIGN}

Developing a HIS adoption roadmap is a challenge thus identification of key HIS technology has to be outlined that enhance quality healthcare. The roadmap framework is based on current HIS technologies, future HIS technologies and the roles stakeholders in healthcare industry. The developed roadmap framework was designed based on the interpreted data and research findings. The different components of the framework were discovered ad confirmed from literature review as some of the factors which affects the adoption of HIS in healthcare institutions.

\subsection{Designing Health Information System Adoption Roadmap}

Based on the findings and expert of health sector engaged, the following framework is developed. The developed roadmap framework was developed from the findings of the research studies. Policy makers and Healthcare-A management could use the presented design to guide HIS adoption. The design shows four factors which affects HIS adoption based on the findings. The design shows the factors which the ministry should consider when adopting HIS, they are; Governance, stakeholder, expert skills development and IS/IT technologies and Infrastructure.

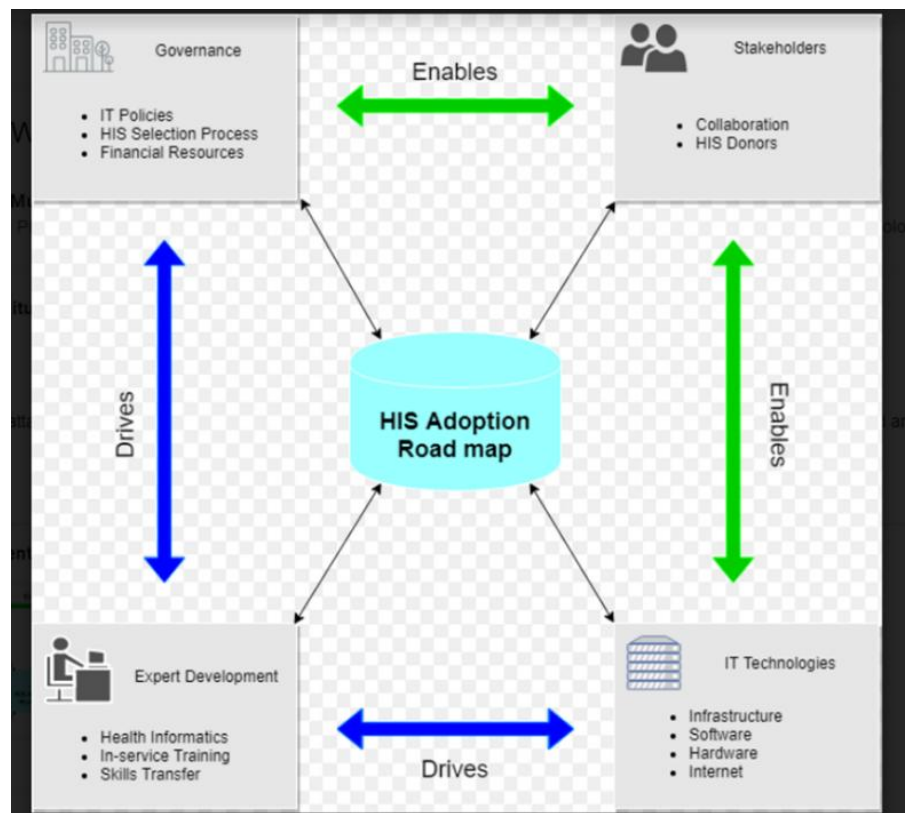

Figure 4. HEALTH Information System adoption roadmap 


\subsubsection{Governance}

Wang and Guo (2015) noted that governance recognises the capacity to get things done without relying on the power of the government to command or use its power. He further emphasised that, in modern society, the government is moving its responsibilities to private sector organisations and NGOs groups, which are undertaking responsibilities that were formerly in the hands of the government. The management and governance of HIS via the IT directors may be an obstacle to the adoption of HIS in the Healthcare. Thus, an employee within the Healthcare-A felt that the process is long and decisions may exclude the users of systems. Again, people dealing with the selection HIS issues may lack knowledge of all systems. As a result, some decisions were not made for the best interest of the whole organisation but rather to serve personal interests. Employees in government health institution feel politics has more power in the development of HIS systems this holds the HIS adoption from the users side. The HIS adoption lacks management support and empowerment from top leadership. Thus, this research finds that proper support is vital during HIS planning and adoption. This concept relates to the non-technical actors, withholding the development of HIS adoption.

\subsubsection{Health Information System Stakeholder}

Stakeholders include government agencies, hospitals, physicians, dentists, nurses, pharmacies, labs, insurers/payers, a variety of associations, and consumer interests (Pradhan, \& Das, 2017). This research records that the Healthcare-A has started with stakeholder engagement in the HIS division. The solo systems were a result of not engaging stakeholders. Until the new directorate was created, there was no single body to lead and coordinate the HIS emphasised one respondent. Stakeholders are actors that will bring and share different ideas toward the enhancement of the HIS and stakeholders are actually those who are most interested in solving the problem (Andriof \& Waddock, 2017).

\subsubsection{Health Information System Technologies}

This concept represents HIS technology that needs to be in place to embark on HIS adoption. Healthcare institutions is affected by a lack of HIS infrastructure because of low resources affecting the budget. It was observed during the interviews that the division of HIS has been moving from different offices and documents were still packed in boxes with other computers. In order for the Healthcare institutions to successfully adopt and yield quality healthcare with HIS. They require proper HIS equipment is such a database, instead of depending on web application systems. The HIS assessment records that the infrastructure for both local and wide area information and communication technology (ICT) for health institutes is either inadequate or very absent, and a lack of required hardware and software. HIS technologies stand between service management and technology to prove repeatable processes and to provide a suitable operating environment for HIS users. Deployment of wireless technologies and mobile technologies to connect HIS with ICT will be a best option for the developing healthcare industries.

\subsubsection{Health Information System Experts Development}

This concept represents qualified experts who can use HIS technologies to enhance quality healthcare. Information technology (IT) has proven an effective instrument when utilised by people with IT knowledge. Advanced technology and people with IT knowledge are key to the utilisation of HIS. The Healthcare-A needs the best IT solutions to manage its HIS. In order to run HIS smoothly the organisation will need to acquire IT infrastructures such as fast computers, client server networks computers, hardware and software suitable for HIS platforms. The framework shows that the government and stakeholder are the enablers of the HIS adoption, the involvement of the stakeholder in developing roadmap for HIS enables Healthcare-A to successfully adopt the HIS.

\section{CONCLUSION}

This paper discussed the advantages of using a roadmap in healthcare. Roadmapping process that involve stakeholders is the approach organisation take to manage high-level and future requirements. Roadmap will help the health sector define their strategic plan at hand by placing the product into focus, it will keep the organisation in the know of the time and costs. The developed roadmap on HIS adoption is only for 
healthcare but it can be appropriate to other industries. The most important outcome is that before the actual roadmap is applied, there is need for more research to be done involving, health sector stakeholders, HIS developers, users and decision makers. This is the reason for focusing on the importance of roadmap in developing healthcare.

\section{REFERENCES}

Andargoli, A. E., Scheepers, H., Rajendran, D., \& Sohal, A. (2017). Health information systems evaluation frameworks: A systematic review. International journal of medical informatics, 97, 195-209.

Andriof, J., \& Waddock, S. (2017). Unfolding stakeholder engagement. In Unfolding stakeholder thinking (pp. 19-42). Routledge.

Beam, A. L., \& Kohane, I. S. (2018). Big data and machine learning in health care. Jama, 319(13), 1317-1318.

Carvalho, M., Fleury, A., \& Lopes, P. (2013). An overview of the literature on technology roadmapping (TRM): Contributions and trends. Technological Forecasting and Social Change, 80(7), 1418-1437.

Cetindamar, D., Phaal, R., \& Probert, D. (2016). Technology management: activities and tools. Macmillan International Higher Education.

Cho, Y., Yoon, S. P., \& Kim, K. S. (2016). An industrial technology roadmap for supporting public R\&D planning. Technological Forecasting and Social Change, 107, 1-12.

Das, L. (2017). Role of Data in Improving Care within a Health System (Doctoral dissertation, PARDEE RAND GRADUATE SCHOOL).

Haines, K. (2018). Professional development for new classroom spaces: extending the concerns-based adoption model.

Li, M. F., \& Feng, J. (2017). Healthcare Road Map to Modernization in Clouds Healthcare Forum for Healthcare Professionals, Medical Device Manufacturers, Pharmaceutical Companies and Average People on Virtual Private Clouds. Accumulated Computer Machinery [ACM], 247-248.

Managements, C. (2014). Roadmapping and related publications. Journal of Innovation and Technology Management, 9(3), 1-19.

Merriam, S. B., \& Grenier, R. S. (Eds.). (2019). Qualitative research in practice: Examples for discussion and analysis. Jossey-Bass.

Pradhan, K., \& Das, R. (2017). Exploration and interpretation of women stakeholders' overall involvement in women led agricultural innovation system (AIS). Indian Research Journal of Extension Education, 17(3), 74-81.

Renals, S., Carletta, J., Edwards, K., Bourlard, H., Garner, P., Popescu-Belis, A., Sabbah, Y. (2014). Roadmap for Conversational Interaction Technologies. Accumulated Computer Machinery [ACM], 39-42.

Tang, A., de Boer, T., \& van Vliet, H. (2011). Building Roadmaps: A Knowledge Sharing Perspective. Accumulated Computer Machinery (ACM), 13-20.

Wang, Q., \& Guo, G. (2015). Yu Keping and Chinese intellectual discourse on good governance. The China Quarterly, 224, 985-1005

Wilbon, A. D. (2012). Interactive planning for strategy development in academic-based cooperative research enterprises. Technology Analysis \& Strategic Management, 24(1), 89-105.

Williams, M. D., Rana, N. P., \& Dwivedi, Y. K. (2015). The unified theory of acceptance and use of technology (UTAUT): a literature review. Journal of Enterprise Information Management, 28(3), 443-488.

Zhang, X., Yu, P., Yan, J., \& Spil, I. T. A. (2015). Using diffusion of innovation theory to understand the factors impacting patient acceptance and use of consumer e-health innovations: a case study in a primary care clinic. BMC health services research, 15(1), 71 . 